\title{
Zwischen Säkularisierung und religiöser Reorganisation - Eine Analyse der Religiosität türkischer und polnischer Neuzuwanderer in Deutschland
}

\author{
Claudia Diehl • Matthias Koenig
}

Zusammenfassung: Dieser Beitrag befasst sich mit der Bedeutung von Religion im Prozess der Integration von Einwanderern in Deutschland. Gestützt auf neueste Daten (SCIP 2010/11) einer Befragung unter polnischen und türkischen Neuzuwanderern wird untersucht, welchen Einfluss das Migrationsereignis als solches auf Religiosität ausübt und wie sich religiöse Partizipation und private religiöse Praxis in der Frühphase der Integration verändern. Die Untersuchung bestätigt, erstens, dass beide Gruppen einen markanten Rückgang religiöser Partizipation erfahren. Dass dieser Rückgang bei muslimischen Türken stärker ausfällt als bei katholischen Polen und sich insbesondere beim Kirchgang oder Moscheebesuch, weniger dagegen bei der privaten Gebetspraxis nachweisen lässt, deutet auf die Bedeutung religiöser Opportunitätsstrukturen hin. Zweitens zeigt sich, dass bei den polnischen Neuzuwanderern der Rückgang an Religiosität mit starken Bindungen in die säkulare deutsche Aufnahmegesellschaft verknüpft ist, während dies bei den türkischen Neuankömmlingen nicht der Fall ist. Nur für jene lässt sich, drittens, beobachten, dass dem anfänglichen Einbruch der Religiosität mit dem Aufenthalt in Deutschland ein allmählicher Wiederanstieg folgt. Die Befunde deuten darauf, dass assimilationstheoretische Argumente um Überlegungen zu Dynamiken symbolischer Grenzziehung zu erweitern sind, die gruppenspezifische Muster des Wandels von Religiosität zu erfassen gestatten. Aufgrund solcher komplexer Dynamiken religiösen Wandels im Migrationsgeschehen ist insgesamt damit zu rechnen, dass öffentlich sichtbare religiöse Diversität ein dauerhaftes Merkmal moderner Einwanderungsgesellschaften darstellen wird.

Schlüsselwörter: Religiosität · Assimilation · Migration · Deutschland

C. Diehl $(\bowtie)$

FB Geschichte und Soziologie, Universität Konstanz,

Postfach 13, 78457 Konstanz, Deutschland

E-Mail: Claudia.Diehl@uni-konstanz.de

\section{Koenig}

Institut für Soziologie, Georg-August-Universität Göttingen,

Platz der Göttinger Sieben 3, 37073 Göttingen, Deutschland

E-Mail: matthias.koenig@sowi.uni-goettingen.de 


\title{
Between secularization and religious reorganization - the religiosity of Polish and Turkish new immigrants in Germany
}

\begin{abstract}
This paper addresses the role of religion in the process of immigrant integration in Germany. Based on novel data (SCIP 2010/11) from a survey among new Polish and Turkish migrants, it particularly focuses on the impact of the migratory event upon religious participation and private religious practice as well as on early trends of changing religiosity in the receiving context. The study confirms, first of all, that both groups of newcomers experience a decrease in religious practices after the migratory event. This decrease is more pronounced among Muslim Turks than among Catholic Poles and more pertinent for worship attendance than for prayer, thus attesting to the relevance of religious opportunity structure. Secondly, it can be shown that among new Polish immigrants, religious decrease is more pronounced among individuals with stronger social ties to the secular German mainstream, while this is not the case for Turks. For them, thirdly, it seems that religious practices are being re-captured after the rather disruptive first couple of months in what may a called a process of religious re-organizations. These group-comparative findings attest to limits of classical assimilation theory and to the relevance of symbolic boundary dynamics. Overall, they underline that publicly visible religious diversity will remain a permanent feature of modern immigrant societies.
\end{abstract}

Keywords: Religiosity $\cdot$ Assimilation $\cdot$ Migration $\cdot$ Germany

\section{Einleitung: Religiosität türkischer und polnischer Neuzuwanderer in Deutschland}

Die Bedeutung von Religion im Migrationsgeschehen erfreut sich zunehmender Aufmerksamkeit sowohl im öffentlichen als auch im sozialwissenschaftlichen Diskurs. Eine lebendige Forschungsliteratur befasst sich mit religiösen Motiven der Migration, mit der religiösen Verarbeitung migrationsbedingter Erfahrungen von Entwurzelung und Exklusion, mit dem Wandel der Religiosität von Migranten und ihren Nachkommen sowie mit der politisch-rechtlichen Regulierung der für gegenwärtige Einwanderungsgesellschaften charakteristischen religiösen Diversität (vgl. umfassend Breton 2012; Cadge und Ecklund 2007). Lag der Fokus der nordamerikanischen Literatur seit den Arbeiten von Oskar Handlin und Will Herberg vor allem auf dem Beitrag von Religionsgemeinschaften für die erfolgreiche Assimilation von Einwanderern und ihren Nachkommen, konzentrierte sich die westeuropäische Literatur vor allem auf die symbolischen und sozialen Grenzen, denen sich Einwanderer aus islamisch geprägten Gesellschaften gegenübersehen (vgl. Foner und Alba 2008). So hat man ethnisch-religiöse Benachteiligungen im Bildungssystem und auf dem Arbeitsmarkt nachgewiesen (vgl. exemplarisch Alba 2005; Adida et al. 2010; Connor und Koenig 2013), hat die von Muslimen vorgebrachten öffentlichen Anerkennungsforderungen dokumentiert (Statham et al. 2005) und die länderspezifischen Varianten der Einbeziehung des Islam in bestehende religionspolitische Arrangements untersucht (Koenig 2007; Laurence 2012).

Im Lichte dieser Kontroversen stellt sich indessen die genuin religionssoziologische Frage, wie die Dynamiken der Religiosität von Einwanderern im weitgehend säkularisierten Kontext Westeuropas zu beschreiben und zu erklären sind. Tatsächlich hat sich die qualitativ ausgerichtete Forschung bereits seit längerem mit individuellen Aneignungs- 
und kollektiven Organisationsformen des Islam befasst und nachgewiesen, dass etliche aus der Religionssoziologie bekannte Phänomene, wie beispielsweise die Individualisierung religiöser Praxis oder die Entstehung post-traditionaler Vergemeinschaftung, auch unter muslimischen Migranten anzutreffen sind (vgl. nur Tezcan 2003; Schiffauer 2004). Quantitative Studien zu muslimischer Religiosität und ihrer Bedeutung im Integrationsprozess sind indessen jüngeren Datums und, nicht zuletzt aufgrund immer noch mangelnder Datenlage, in ihren Befunden bislang alles andere als eindeutig (vgl. Voas und Fleischmann 2012). Manche Studien in Frankreich (Tribalat 1995), Belgien (Smits et al. 2010) und in den Niederlanden (Maliepaard et al. 2010; Phalet et al. 2008) weisen auf eine nachlassende Bindungskraft des Islam von der ersten zur zweiten Generation hin. Andere Studien kommen dagegen zu dem Ergebnis einer größeren inter-generationalen Stabilität von Religiosität unter Muslimen (für Deutschland vgl. Diehl und Koenig 2009; Jacob und Kalter 2013). Wieder andere Studien betonen die Kontextabhängigkeit religiöser Dynamiken und verweisen dazu auf direkte Effekte xenophober Einstellungen in der lokalen Umwelt auf muslimische Religiosität (Connor 2010) oder auf vermittelnde Effekte des Ausmaßes institutioneller Anerkennung des Islam auf den Zusammenhang von muslimischer Religiosität und sozialer oder struktureller Integration (Fleischmann und Phalet 2012).

Dass etliche Fragen zur Religiosität von Einwanderern in Westeuropa bislang ungeklärt bleiben, ist indessen nicht allein der Datenlage zuzuschreiben, sondern hat auch mit grundsätzlichen Orientierungen der Forschung zu tun. Das wohl wichtigste Defizit sowohl der qualitativen als auch der quantitativen Literatur ist darin zu sehen, dass sie es bislang weitgehend versäumt hat, die religiöse Dynamik von Muslimen mit derjenigen anderer Einwanderergruppen zu vergleichen, insbesondere von Christen, die gegenwärtig immerhin etwa $60 \%$ der nach Europa kommenden Einwanderer ausmachen (Pew Research Centre 2012, S. 52). Die wenigen Survey-basierten Studien, die die Integrationsmuster verschiedener religiöser Einwanderergruppen in Europa vergleichen (Aleksysnka und Algan 2010, S. 27; Van Tubergen und Sridottir 2011) leiden häufig an kleinen Fallzahlen und unterkomplexen Messinstrumenten von Religiosität. Dass indessen eine gruppenvergleichende Betrachtung für das allgemeine Verständnis religiöser Dynamiken im Migrationsgeschehen erforderlich ist, dürfte auf der Hand liegen. Eine weitere Begrenzung der bisherigen Forschung ist darin zu sehen, dass sie die komplexen Prozesse des Wandels von Religiosität bei Einwanderern im Kohorten-, Generationen- und Zeitverlauf nur unpräzise erfasst und die dabei wirksamen Mechanismen ungeklärt lässt (vgl. in diesem Zusammenhang auch Stolz 2010). Praktisch gar nichts lässt sich bislang beispielsweise über die Anfangsphase der Integration von Einwanderern sagen, obwohl sie für längerfristige Dynamiken sozialer und struktureller Assimilation, ethnischer Gruppenbildung und eben auch religiösen Wandels von weichenstellender Bedeutung sein dürfte. Während der Einfluss des Migrationsereignisses auf Religiosität im Kontext der USA und Kanadas inzwischen sorgfältig untersucht wurde, gab es bis vor kurzem keine Daten, die es erlaubt hätten, diese frühen Integrationsdynamiken auch in Westeuropa zu untersuchen.

In diesem Aufsatz leisten wir einen Beitrag zum Verständnis der Bedeutung von Religion im Migrationsgeschehen, indem wir neueste Daten des Forschungsprojekts Socio-Cultural Integration Processes of New Immigrants in Europe (SCIP) präsentie- 
ren. Dessen Befragungsinstrument gestattet es, Veränderungen religiöser Praktiken in der Frühphase des Integrationsprozesses zu beleuchten. Wir konzentrieren uns auf zwei Kategorien von Neuzuwanderern: muslimische Türken und katholische Polen in Deutschland. Beide stammen aus hochreligiösen Herkunftsländern und teilen damit die Erfahrung des abrupten Wechsels in eine hochsäkularisierte Gesellschaft. Beide unterscheiden sich allerdings darin, dass sie sich unterschiedlichen religiösen Feldern und symbolischen Grenzen gegenübersehen. Ein Vergleich beider Gruppen vermag daher aufschlussreiche Einsichten in die facettenreiche Dynamik religiösen Wandels unmittelbar nach dem Migrationsereignis zu eröffnen.

Zunächst skizzieren wir einige theoretische Argumente über mögliche Dynamiken der Religiosität bei Neuzuwanderern (Abschn. 2). Wir liefern im nächsten Schritt einige Hintergrundinformationen über die türkische und polnische Einwanderungsgeschichte in Deutschland und formulieren die sich daraus ergebenden Erwartungen für den frühen religiösen Anpassungsprozess (Abschn. 3). Nach einer knappen Vorstellung des deutschen SCIP-Datensatzes (Abschn. 4) präsentieren wir erste empirische Befunde, die zeigen, dass die Häufigkeit des Besuchs religiöser Veranstaltungen wie auch des Gebets bei beiden Gruppen zunächst abrupt abnimmt, mit dem längerem Aufenthalt aber unterschiedlichen Dynamiken ausgesetzt ist (Abschn. 5). Bei den Polen scheint der anfängliche, vermutlich auf mangelnde Opportunitäten zurückgehende Rückgang religiöser Partizipation den Beginn einer Anpassung an den säkularen Mainstream darzustellen, während ihm bei den Türken unabhängig von sonstigen Integrationsdimensionen ein allmählicher Wiederanstieg von Religiosität auf ihr ursprüngliches Niveau folgt.

\section{Theoretische Argumente}

Über den Wandel der Religiosität bei Neuzuwanderern wird in der nordamerikanischen Literatur bereits seit längerem diskutiert. Dabei bietet es sich an, zwei distinkte Teilfragen voneinander zu unterscheiden. Einerseits ist nach den Effekten des Migrationsereignisses auf die Religiosität von Einwanderern im Vergleich zu deren im Herkunftsland gepflegten Gewohnheiten, andererseits nach den bei andauerndem Aufenthalt im Aufnahmeland einsetzenden Veränderungsprozessen der Religiosität zu fragen. In beiderlei Hinsicht ist überdies in Rechnung zu stellen, dass religiöse Trends und ihr Zusammenhang mit anderen, etwa sozialen oder strukturellen Dimensionen der Integration, zwischen verschiedenen Gruppen variieren können. Im Folgenden diskutieren wir beide Fragen in Anlehnung an theoretische Überlegungen aus der Migrations- und Religionssoziologie und unter Rückgriff auf bisherige empirische Befunde aus Nordamerika. Drei Hauptargumente sind es, die sich für den Wandel von Religiosität in der hochdynamischen Anfangsphase der Integration von Einwanderern formulieren lassen.

Was die Effekte des Migrationsereignisses selbst angeht, so meinte man lange, die Migration sei eine traumatische Erfahrung, die als solche bereits eine stärkere Hinwendung zur Religion auslösen könne. Sie stelle gewissermaßen ein ,theologisierendes“ Ereignis dar, in welchem Wanderung in religiösen Semantiken von „Exodus“, „Pilgerschaft" und Ähnlichem gedeutet würden (so v. a. Smith 1978, S 1173). Organisierte Religionsgemeinschaften böten den Einwanderern „refuge, respectability and resources“ 
(Hirschman 2004) und entsprechend sei, wie die klassischen Schilderungen der irischen, italienischen und polnischen Zuwanderung in den USA zeigten, die Gründung ,ethnischer Kirchen“ eine der ersten Aktivitäten neuer Zuwanderergruppen (vgl. Mullins 1987 sowie neuerdings Alba et al. 2009). Unterstellt wurde oftmals ein Mechanismus, wie er aus religionssoziologischen Säkularisierungstheorien geläufig ist (vgl. etwa Norris und Inglehart 2004). Religiosität sei eine Antwort auf Erfahrungen von Kontingenz oder existenzieller Unsicherheit, und insoweit die mit einer Migrationsentscheidung typisch verbundene Entwurzelung solche Erfahrungen verstärke, erhöhe sie die Nachfrage nach religiösen Sinn- und Deutungsangeboten.

Trotz ihrer prima facie-Plausibilität konnten diese Überlegungen in neueren empirischen Studien indessen bislang gerade nicht bestätigt werden. Repräsentative Studien unter Neuzuwanderern in den USA und in Kanada zeigen vielmehr durchgehend, dass Standardindikatoren religiöser Partizipation, beispielsweise der Gottesdienstbesuch, nach der Migration zunächst ab- und nicht zunehmen (Cadge und Ecklund 2006; Connor 2008, 2009a, b; Massey und Higgins 2011; eine Übersicht älterer Studien findet sich bei Mol 1979, S. 33). Zwar mag es sein, dass gerade die ersten Einwandererkohorten hochgradig religiös aktiv sind; bei nachfolgenden Kohorten scheinen sich indessen andere religiöse Dynamiken abzuspielen.

Ein erstes Argument, das in der aktuellen Diskussion vertreten wird, greift daher auf Einsichten der religionssoziologischen Angebotstheorie zurück, die religiöse Partizipation generell auf vorhandene Opportunitätsstrukturen zurückführt. Dass Migration zunächst einen Rückgang gemeinschaftlich praktizierter Religiosität auslöst, kann aus dieser Sichtweise nicht verwundern. In den alltäglichen Handlungsentscheidungen von Einwanderern stehen religiöse Aktivitäten in Konkurrenz mit kurzfristig dringlicher erscheinenden Aktivitäten, angefangen von Wohnungs- und Jobsuche über den Spracherwerb bis hin zum Aufbau sozialer Kontakte (zum theoretischen Hintergrund vgl. Stolz 2009 und Stolz in diesem Band). Neuzuwanderern fehlt nicht nur die Zeit, ihnen fehlt auch (Information über) die religiöse Infrastruktur im Aufnahmeland, was sich in einem Rückgang religiöser Partizipation (nicht unbedingt aber privater religiöser Praxis und Überzeugungen) äußern dürfte. Damit ist gleichzeitig auch gesagt, dass mit längerer Aufenthaltsdauer religiöse Partizipation auf ihr vorangegangenes Niveaus wiederansteigen sollte. Aus diesem allgemeinen Argument lassen sich ferner auch gruppenspezifische Implikationen ableiten. Insbesondere Angehörigen religiöser Minderheiten dürfte Zugang zu oder Wissen über Kirchen, Moscheen und Tempel fehlen, sodass bei ihnen der Rückgang religiöser Partizipation stärker ausgeprägt sein sollte als bei Angehörigen religiöser Mehrheiten; tatsächlich scheint beispielsweise im katholisch geprägten Quebec der Gottesdienstbesuch bei katholischen Neuzuwanderern im Vergleich zu anderen etwas stabiler zu sein (vgl. Connor 2008). Schließlich könnte man vermuten, dass der anfängliche Rückgang religiöser Partizipation in Kontexten mit einem hohen Grad religiöser Diversität und entsprechender Angebotsvielfalt weniger scharf ausfällt (so für Nordamerika Connor 2009a).

Was aber passiert mit fortschreitendem Aufenthalt im Aufnahmeland? Ist der soeben genannte Rückgang religiöser Partizipation nach dem Migrationsereignis eine temporäre Abweichung von habituellen Mustern, die sich später wieder stabilisieren, oder ist er der Beginn einer längerfristigen Anpassung an die Mehrheitsgesellschaft? Im 
nordamerikanischen Kontext lassen sich diese beiden Szenarien nur schwer voneinander scheiden, da die Anpassung an die nach wie vor hochreligiöse Mehrheitsgesellschaft eine Stabilisierung religiöser Partizipation auf hohem Niveau erwarten ließe; tatsächlich deuten einzelne Studien zu Konversionen, denominationalem ,switching“ und Kirchgangshäufigkeit in genau diese Richtung (vgl. Akresh 2011). Umso interessanter ist es, sie im europäischen Kontext zu diskutieren, wo (spätestens) seit den 1960er Jahren dramatische Säkularisierungsprozesse stattgefunden haben (vgl. nur Voas 2009). Ausgangspunkt dafür ist die neuere migrationssoziologische Assimilationstheorie, in deren Perspektive die frühe Disruption der Religiosität von Neuzuwanderern als Anfangsepisode eines längeren Prozesses sozialer, struktureller und kultureller Integration erscheint.

Das zweite Argument knüpft entsprechend an die Assimilationstheorie an und unterstellt (in Europa) eine allmähliche Assimilation von Einwanderern an den säkularen Mainstream (vgl. dazu auch Diehl und Koenig 2009, S. 302). Dabei wird das Verhalten von Einwanderern generell als Investitionsstrategie modelliert, bei der die Individuen zwischen ethnischen und mehrheitsbezogenen Handlungsoptionen entscheiden (vgl. z. B. Esser 2006; ähnlich schon Alba und Nee 2003). So lange neu zugewanderte Einwanderer ihren Aufenthalt im Aufnahmeland als temporär betrachten, haben sie starke Anreize dafür, in ethnische Bindungen und Orientierungen zu investieren, da diese als relevant für die erwartete Rückkehr ins Herkunftsland angesehen werden. Steigt die Bleibeabsicht, so werden diese Anreize schwächer und es treten aufnahmelandbezogene Optionen in den Vordergrund, wie beispielsweise der Spracherwerb, Bildung, Berufseinstieg oder Freundschaftsnetzwerke. Religiöse Praktiken werden auf dieser Argumentationslinie typischerweise als Investition in ethnische Orientierungen interpretiert. Dabei kann man sich auf Bretons (1964) Analyse ethnischer institutioneller Vollständigkeit (,,institutional completeness“") stützen, der zufolge die Einbindung in religiöse Organisationen ein wirksamer Mechanismus zur Pflege ethnischer Netzwerke ist; auch für den Aufrechterhalt transnationaler Bindungen hat die qualitative Forschung religiöse Organisationen als hochrelevant eingestuft (vgl. z. B. Hagan und Ebaugh 2002; Levitt 2003).

Auf der Individualebene wäre aus dieser Perspektive zu erwarten, dass Religiosität bei denjenigen rückläufig ist, die sich bereits deutlich von ethnischen Orientierungen distanziert haben und in struktureller wie sozialer Hinsicht in die Aufnahmegesellschaft integriert sind. Auf der Gruppenebene ließen sich ebenfalls Unterschiede erwarten. Gruppenmerkmale, die sich auf individuelle Strategien direkt auswirken, sind Esser (2006) zufolge etwa die Gruppengröße, die institutionelle Vollständigkeit und ethnische Grenzziehungen. Angehörige von Migrantengruppen, die sich im öffentlichen Diskurs und/oder in institutionellen Arrangements der Religionspolitik salienten symbolischen Grenzen gegenübersehen, hätten demzufolge mit hohen Kosten zu rechnen, wenn sie ethnische Bindungen und damit auch religiöse Praktiken aufrechterhielten (vgl. auch Alba 2005). Bei gleicher sozialer oder struktureller Assimilation müsste man bei ihnen mit einem stärkeren Rückgang religiöser Praktiken rechnen als bei Neuzuwanderern, die unscharfe (,,blurred“) symbolische Grenzen antreffen. Für diese wären „bikulturelle“ Optionen (Berry 1997), also Orientierungen sowohl an der eigenen ethnischen Gruppe als auch an 
der Aufnahmegesellschaft möglich, was sich moderierend auf den Zusammenhang von Assimilationsindikatoren und Religiosität auswirken müsste. ${ }^{1}$

Die soweit skizzierte Assimilationstheorie ist indessen, gerade was ihre religionssoziologischen Annahmen angeht, nicht frei von Problemen. Strittig ist insbesondere, ob Religiosität schlechterdings als ethnische Investitionsstrategie verbucht werden kann. Zwar ist es richtig, dass Religiosität ein Ausdruck ethnischer Identität sein kann. Religionssoziologische Überlegungen zum „,cultural defense“ haben darauf mit Blick auf die Religiosität von Einwanderern und Angehörigen peripherer Nationen vielfach hingewiesen (Bruce 2011). Aber ebenso richtig ist, dass religiöse Praktiken sich von Ethnizität entkoppeln können (vgl. Abramson 1979; Hammond und Warner 1993; Green 2002; Mitchell 2006). Dies war bereits die Pointe von Herbergs (1956) klassischer Analyse der Ablösung herkunftslandbezogener durch konfessionelle Identitäten im Generationenverlauf der europäischen Einwanderer in den USA (vgl. auch Gordon 1964), und Mullins (1987) hat sie in seiner Analyse der Transformation von ethnischen in multi-ethnische Kirchen für die Organisationsebene reformuliert. Tatsächlich gibt es auch im westeuropäischen Kontext etliche Hinweise auf eine solche De-Ethnisierung der Religion von Einwanderern. Studien zur Veränderung religiöser Organisationen im deutschen Islam (Schiffauer 2009) deuten ebenso in diese Richtung wie Befunde empirischer Studien, denen zufolge die Religiosität muslimischer Einwanderer vergleichsweise stabil ist, gleich ob sie in ethnisch segregierten oder gemischten Nachbarschaften leben (Bisin et al. 2008). Während die Stabilität im Generationenverlauf (Diehl und Koenig 2009; Jacob und Kalter 2013) auf die Bedeutung religiöser Sozialisation im Familienkontext verweist, deutet die Beständigkeit religiöser Praktiken bei der ersten Generation der Einwanderer darauf hin, dass sich tief verankerte Werte und Identitäten langsamer verändern als kognitive Wissensbestände, soziale Netzwerke und strukturelle Positionen (so Phalet und Schönpflug 2001).

Angesichts dieser Anfragen an die Assimilationstheorie schlagen wir ein drittes Argument vor, das religiöse Grenzziehungskonfigurationen und gruppenspezifische religiöse Dynamiken in den Vordergrund rückt (vgl. auch Diehl und Koenig 2009, S. 315). Für Neuzuwanderer, die sich scharfen religiösen Grenzen gegenübersehen, ließe sich erwarten, dass das Migrationsereignis und die Aufenthaltsdauer die Salienz zuvor eher impliziter Identitäten verstärken kann (Hardin 2001). Angesichts der Stigmatisierung des Islams wäre insbesondere bei Muslimen mit der Persistenz religiöser Praktiken zu rechnen, ohne dass dies notwendigerweise mit „ethnischen“ Orientierungen einhergehen müsste. Auf dieser Argumentationslinie würde man erwarten, dass nach der anfänglichen Phase des Einbruchs religiöser Praxis die vorherigen Niveaus allmählich wieder erreicht werden. Vor allem aber wäre zu erwarten, dass diese Form religiöser Reorganisation von anderen Dimensionen der Assimilation weitgehend unabhängig ist. Anders als dem zweiten Argument zufolge, erschiene die Entkopplung von religiöser und sozialer oder struktureller Assimilation für Einwanderergruppen, die salienten religiösen Grenzen gegenüberstehen, dann als wahrscheinlicher, nicht als unwahrscheinlicher.

1 Dies könnte auch zur Entstehung von ,symbolischer Religiosität“ (Gans 1994) führen, die allerdings, wenn überhaupt, erst bei langfristigem Aufenthalt oder im Generationenverlauf nachzuweisen sein dürfte (vgl. Diehl und Koenig 2009, S. 303). 
Zusammenfassend lässt sich festhalten, dass die Religiosität von Neuzuwanderern sowohl von Effekten des Migrationsereignisses als auch von bereits einsetzenden Integrationsprozessen geprägt ist. Das Migrationsereignis, so unser erstes Argument, wirkt sich aufgrund der im Aufnahmeland fehlenden Opportunitäten zunächst disruptiv auf organisierte Religiosität aus, insbesondere bei religiösen Minderheiten. Parallel zu sozialer und struktureller Assimilation, so unser zweites Argument, setzen sodann Mechanismen religiöser Adaptation ein, die in einem säkularen Integrationskontext zu einem Rückgang an Religiosität führen. Diese Mechanismen, so unser drittes Argument, greifen indessen nicht für alle Gruppen gleichermaßen; bei religiösen Minderheiten, die salienten symbolischen Grenzen gegenüberstehen, kann es vielmehr zu einer Re-Organisation von religiösen Aktivitäten kommen.

\section{Polen und Türken in Deutschland}

Für eine gruppenvergleichende Überprüfung der genannten theoretischen Argumente eignet sich der westeuropäische Integrationskontext in besonderer Weise. Denn nicht nur ist er von den bereits erwähnten Säkularisierungsprozessen gekennzeichnet, ihn charakterisieren gleichzeitig scharfe symbolische Grenzen gegenüber dem Islam, die sich in öffentlichen Diskursen und Einstellungen ebenso niederschlagen wie im Fortbestehen historisch begründeter rechtlicher Privilegien für christliche Kirchen. In unserer empirischen Analyse zur Religiosität von Neuzuwanderern konzentrieren wir uns auf muslimische Türken und katholische Polen, die kürzlich nach Deutschland gekommen sind. Die wichtigsten Hintergrundinformationen zu beiden Bevölkerungsgruppen fassen wir im Folgenden zusammen, um auf dieser Grundlage die allgemeinen theoretischen Argumente in spezifische Hypothesen zu überführen.

Die gegenwärtige Zuwanderung aus der Türkei und Polen fügt sich in je spezifischer Weise in die komplexe Migrationsgeschichte der bundesdeutschen Nachkriegszeit ein. Die inzwischen etwa 2,8 Millionen. Einwohner mit türkischem Migrationshintergrund stellen die zahlenmäßig größte nicht-deutsche Einwanderungsgruppe in Deutschland dar. Die Pioniergeneration bestand aus vorwiegend männlichen, niedrig qualifizierten Arbeitsmigranten, die in den 1950er und 1960er Jahren kamen, um den Arbeitskräftebedarf der bundesdeutschen Wirtschaft zu decken. Nach dem Anwerbestopp (1973) kam es, dank europäischer rechtlicher Regelungen und angesichts politischer Konflikte in der Türkei, zu vermehrtem Familiennachzug. Bis heute stellt der Familiennachzug das wichtigste Migrationsmotiv für Zuzüge aus der Türkei dar (Migrationsbericht 2008, S. 206). Es nehmen zudem einige jüngere türkische Zuwanderer ein Studium in Deutschland auf, und im Rahmen der (bislang zögerlichen) deutschen Beteiligung am globalen Wettbewerb um Fachkräfte ist auch eine kleine Zahl hochqualifizierter türkischer Arbeitsmigranten eingewandert. Insgesamt aber sind die Einwanderungszahlen aus der Türkei seit Jahren auf ohnehin schon niedrigem Niveau rückläufig.

Ganz anders stellt sich die Situation der polnischen Einwanderung dar. Klammert man die historischen Zuzüge polnischsprachiger Landarbeiter aus den ostpreußischen Provinzen des Deutschen Kaiserreichs in die industriellen Zentren des Westens aus, so sind die inzwischen etwa 640000 Einwohner mit polnischem Migrationshintergrund 
hauptsächlich nach dem Fall des sozialistischen Regimes gekommen, vorwiegend als Spätaussiedler, Saisonarbeiter oder Studierende (Migrationsbericht 2008, S. 37-38). Mit dem Beitritt Polens zur Europäischen Union (2004) haben polnische Arbeitnehmer neue Rechte auf Freizügigkeit auf dem europäischen Arbeitsmarkt erhalten, wobei die Bundesrepublik jene Rechte bis Mai 2011 temporär einschränkte.

Beide Herkunftsländer, die sunnitisch geprägte Türkei und das katholische Polen, stechen im innereuropäischen Vergleich als religiös ausgesprochen homogene und gleichzeitig vitale Gesellschaften heraus. Dem International Social Survey Programme (ISSP 2008) zufolge bezeichnen sich jeweils mehr als drei Viertel der türkischen und polnischen Bevölkerung als etwas, sehr oder extrem religiös; und die Zahlen für den mindestens monatlichen Kirch- oder Moscheegang liegen in beiden Ländern bei etwa 50\%. Beide Länder können überdies als Beispiele für die von Casanova (1994) beschriebene „Deprivatisierung" von Religion gelten. Nationalistische Bewegungen, die auf islamische oder katholische Identitäten Bezug nehmen sowie demokratische Protestbewegungen gegen den Kemalismus oder gegen den Sozialismus haben Religion in der öffentlichen Sphäre beider Länder gleichermaßen sichtbar werden lassen (vgl. beispielsweise Yavuz 2003; Zubrzycki 2006). Für Einwanderer aus beiden Ländern muss man daher davon ausgehen, dass der Zuzug nach Deutschland oder in andere westeuropäische Gesellschaften, in denen Religion an gesellschaftlicher Bedeutung eingebüßt hat, weitreichende Konsequenzen für religiöse Identitäten, Glaubensüberzeugungen und Praktiken hat.

Diese Konsequenzen dürften sich, folgt man den oben eingeführten theoretischen Argumenten, in mehrfacher Hinsicht unterscheiden. Was, erstens, das Argument religiöser Opportunitäten angeht, ist zwar zunächst bei beiden Gruppen ein Rückgang religiöser Partizipation zu erwarten. Es ist aber in Rechnung zu stellen, dass Türken und Polen unterschiedlich strukturierte religiöse Subfelder in Deutschland vorfinden. Angesichts zahlenmäßiger Größe und organisatorischer Ressourcen der katholischen Kirche dürften polnische Einwanderer recht schnell auf ausgebildete religiöse Opportunitätsstrukturen treffen. Zwar ist es richtig, dass sich in Deutschland ein lebendiges Feld muslimischer Vereinigungen mit starkem Wettbewerb zwischen den verschiedenen Strömungen des Islam entwickelt hat, insbesondere zwischen den dem türkischem Staatsislam nahe stehenden Moscheevereinen der „Diyanet“ und den Moscheevereinen des oppositionellen politischen Islam (Milli Görüs, Süleymanci, Gülen Bewegung). Doch trotz politischer Initiativen zur Gründung von Dachverbänden, wie beispielsweise in der Deutschen Islamkonferenz (vgl. dazu Laurence 2012; Schiffauer 2009), bleiben etliche administrative Probleme des organisierten islamischen Lebens, von der Anerkennung als Körperschaft des öffentlichen Rechts ganz zu schweigen (vgl. dazu Joppke in diesem Band), ungelöst. Verglichen zum Katholizismus stellt sich das organisierte religiöse Angebot für muslimische Einwanderer als ungünstig dar. Die angebotsbedingten Rückgänge religiöser Partizipation sollten bei türkischen Neuzuwanderern daher schärfer ausfallen als bei polnischen.

Das zweite, assimilationstheoretische Argument behauptet, dass sich ein dauerhafter Rückgang von Religiosität insbesondere bei denjenigen Einwanderern beobachten lässt, die starke aufnahmelandbezogene Orientierungen aufweisen, die also beispielsweise in Deutschland zu bleiben beabsichtigen, starke soziale Bindungen in die Mehrheitsgesellschaft aufbauen und in ihr auch strukturell integriert sind. Stabil müsste Religiosität 
demgegenüber sein, wenn Einwanderer eine starke Rückkehrorientierung haben, strukturell weniger integriert sind, in segregierten Nachbarschaften leben und ethnische soziale Kontakte pflegen. In gruppenvergleichender Perspektive würde man vermuten, dass die Stärke dieser Zusammenhänge variiert, weil Polen und Türken sich hinsichtlich ethnischer Gruppengröße, institutioneller Vollständigkeit und symbolischen Grenzen deutlich unterscheiden. Insbesondere in Großstädten wie Berlin oder Köln dürften türkische Neuzuwanderer auf institutionell verfestigte ethnische Netzwerke treffen, während dies bei polnischen Neuzuwanderern kaum zutrifft. Außerdem sind sie mit größeren sozialen Distanzen seitens der einheimischen Bevölkerung konfrontiert und fühlen sich häufiger diskriminiert (Blohm und Wasmer 2008; Hans 2010). Folgt man der Assimilationstheorie, so müsste man also annehmen, dass gerade bei ihnen eine frühe strukturelle und soziale Assimilation mit einem deutlichen Rückgang an Religiosität einhergeht; der Aufrechterhalt muslimischer religiöser Praktiken bedeutete, zumal angesichts der symbolischen Grenzen gegenüber dem Islam, zu hohe Investitionskosten.

Das dritte Argument setzt demgegenüber etwas andere Akzente. Die Salienz der religiösen Grenze gegenüber dem Islam würde man hier genau umgekehrt als günstige Bedingung für die Aufrechterhaltung religiöser Gewohnheiten betrachten, und zwar unabhängig von frühen Assimilationsprozessen in anderen Dimensionen. Der Aufbau sozialer Bindungen mit Deutschen würde ebenso wie die Partizipation im deutschen Arbeitsmarkt aus dieser Sicht allenfalls für die Polen mit einer Anpassung an die säkulare Mehrheitskultur einhergehen. Muslimische Türken können sich in religiöser Hinsicht nicht allmählich an die Mehrheitskultur anpassen, weil ihnen in jedem Falle die Fremdzuschreibung als ,Muslim“ entgegengebracht wird. Religiöse Identitäten könnten in einem Wechselspiel von Fremd- und Selbstzuschreibung verfestigt oder gar verstärkt werden, selbst wenn es zur Assimilation in anderen Bereichen kommt. Der abrupte Einbruch religiöser Praxis nach dem Migrationsakt wäre für muslimische Türken also eine temporäre Episode, die keinesfalls eine spätere Re-Organisation religiöser Praktiken ausschließt, während sie bei Polen durchaus den Anfang eines längeren religiösen Assimilationsprozesses darstellen könnte.

\section{Daten und Methoden}

Um die soweit entfalteten Argumente und Hypothesen zur Religiosität polnischer und türkischer Neuzuwanderer zu testen, verwenden wir Survey-Daten, die in dem internationalen Projekt Socio-Cultural Integration Processes among New Immigrants in Europe (SCIP) erhoben wurden. ${ }^{2}$ Der Survey wurde als Mini-Panel mit zwei Erhebungswellen unter ausgewählten Migrantengruppen in vier europäischen Zielländern (Deutschland, die Niederlande, Großbritannien, Irland) durchgeführt. Insgesamt wurden mehr als 7000 Neuzuwanderer im Alter von 18 bis 60 Jahren in ihrer jeweiligen Herkunftssprache befragt. In diesem Artikel konzentrieren wir uns auf Daten der ersten, 2010/11 durchgeführten Erhebungswelle in Deutschland. In türkischen oder polnischen CAPI-Interviews wurden Neuzuwanderer befragt, die vor maximal eineinhalb Jahren nach Deutschland

2 Das in vier Ländern durchgeführte SCIP Projekt wird vom NORFACE Research Programme on Migration finanziert und von Claudia Diehl an der Universität Konstanz koordiniert. 
zugezogen waren. Zufallsstichproben der beiden Gruppen wurden aus Bevölkerungsregistern von fünf deutschen Großstädten (Berlin, Bremen, Hamburg, Köln und München) gezogen. Letztlich konnten in der ersten Erhebungswelle 2697 face-to-face-Interviews (1516 Polen; 1181 Türken) geführt werden. Unsere Analyse beschränkt sich auf diejenigen, die der jeweiligen Mehrheitsreligion angehören. Nach Ausschluss der wenigen Polen und Türken, die keiner oder einer anderen als der Mehrheitsreligion anzugehören angeben, verbleiben 1343 katholische Polen und 1028 muslimische Türken in unserem Sample.

Der SCIP-Fragebogen umfasst eine Reihe klassischer Items, die in der Religionssoziologie zur Messung des multidimensionalen Konzepts von Religiosität (Glock 1962) verwendet werden: Kirchgang oder Moscheebesuch, Gebetshäufigkeit, Fasten, das Tragen des Kopftuchs (bei Musliminnen), die Selbsteinschätzung der eigenen Religiosität und die relative Stärke der Identifikation mit Christentum oder Islam. ${ }^{3}$ Zudem werden für diejenigen, die regelmäßig die Kirche oder Moschee besuchen, Informationen zur ethnischen Zusammensetzung ihrer religiösen Gemeinden, zu deren eventueller Hilfestellung bei Alltagsproblemen, wie Wohnungs- und Jobsuche, und zu Erfahrungen religiöser Diskriminierung erhoben. Diese Vielfalt an Informationen nutzen wir im Folgenden zur Interpretation der Ergebnisse unserer Analyse. Im Zentrum stehen allerdings Veränderungen der Religiosität nach dem Migrationsereignis.

In unserer Analyse verwenden wir daher diejenigen Variablen, die für beide Gruppen auch retrospektiv über die Zeit vor der Migration vorliegen, nämlich Kirchgang bzw. Moscheebesuch und Gebet. ${ }^{4}$ Die daran abzulesenden Veränderungen religiöser Partizipation und privater religiöser Praxis nach dem Migrationsereignis stellen wir im ersten Schritt unserer Analyse deskriptiv dar. Im zweiten Schritt gehen wir zu einer multivariaten Betrachtung über, die unsere theoretischen Argumente und Hypothesen zu überprüfen gestattet. In unserer Modellierung orientieren wir uns an den Strategien ähnlicher Studien aus Nordamerika. Manche dieser Studien behandeln die $\mathrm{Zu}$ - oder Abnahme von Religiosität direkt als abhängige Variable (Connor 2008, S. 252, 2009, S. 793), verlieren damit aber Niveauunterschiede aus dem Blick. Wir folgen daher diejenigen Studien, die Religiosität nach der Migration als abhängige Variable behandeln und unter den multivariat analysierten unabhängigen Variablen auch die Religiosität vor der Migration berück-

3 Aus den für beide Gruppen abgefragten Items lässt sich, wie separate (hier nicht dargestellte) Analysen zeigen, ein additiver Religiositätsindex bilden (Cronbachs Alpha=0,746), der aufgrund unseres Interesse an dem Vergleich der Religiosität vor und nach der Migration im Folgenden jedoch nicht Verwendung findet.

4 Zwar wurden retrospektive Fragen auch zu religiösem Fasten und (bei Türkinnen) zum Tragen des Kopftuchs gestellt. Da einige der Befragten aufgrund ihres kurzen Aufenthalts noch keine Gelegenheit zum Fasten während der Passionszeit oder des Ramadan hatten und wir an Gruppenvergleichen interessiert sind, klammern wir beide Fragen aus der eigentlichen Analyse aus. Freilich sind auch die von uns verwendeten Retrospektivangaben nicht ganz unproblematisch. Erstens könnte es sein, dass diese aufgrund der Wahrnehmung kontextueller Unterschiede auch die eigene Religiosität überschätzen, und zweitens ist denkbar, dass die Migration mit Lebenslaufübergängen (etwa der Geburt von Kindern) zusammenfällt, die ohnehin zu einer Veränderung religiöser Praxis geführt hätten. Auch wenn wir beide Einwände nicht endgültig entkräften können, halten wir das, auch in der bisherigen Literatur verwendete Vorgehen gerade für Gruppenvergleiche für tragfähig. 
sichtigen (Akresh 2011, S. 654; Massey und Higgins 2011, S. 1385). Diese Strategie hat den Vorteil, dass sie die primär interessierende Religiosität nach der Migration ins Zentrum der Analyse stellt. Dabei wird angenommen, dass Kirchgang oder Moscheebesuch und Gebet in hohem Maße habituell verankert und damit von den jeweiligen Niveaus vor der Migration bestimmt sind, dass die verbleibende Varianz aber durch Variablen erklärt werden kann, die unsere eingangs eingeführten theoretischen Argumente erfassen.

Wir beginnen mit einem Basismodell (Modell I), das zunächst die sozio-demographischen Variablen Geschlecht, Alter, Bildung und regionaler Hintergrund enthält, die in der Religionssoziologie vielfach als allgemeine Determinanten von Religiosität angesehen werden. Das Basismodell enthält ferner die retrospektiven Angaben zu religiösen Praktiken, um der Frage nach den Effekten des Migrationsereignisses nachzugehen. Es enthält schließlich auch die Aufenthaltsdauer (in Monaten), um die Trends religiöser Praktiken nach Ankunft in Deutschland zu erfassen. ${ }^{5}$ Das Basismodell schätzen wir für beide Gruppen separat. Dadurch, sowie durch den Vergleich der Resultate für Kirchgang oder Moscheebesuch einerseits und Gebet andererseits, kann das erste, auf religiöse Angebotsstrukturen abzielende Argument geprüft werden.

Um das zweite, der Assimilationstheorie entlehnte Argument zu überprüfen, erweitern wir das Basismodell im nächsten Schritt um zusätzliche theoretisch relevante Variablen (Modell II). Zunächst berücksichtigen wir die Migrationspläne der Neuzuwanderer; diejenigen, die in Deutschland zu bleiben beabsichtigen, dürften stärker auf die Aufnahmegesellschaft hin orientiert sein als diejenigen, die bereits die Rückkehr in ihr Herkunftsland, dauerhaftes Pendeln oder die Wanderung in ein drittes Land planen. Um strukturelle Assimilation zu erfassen, ergänzen wir ferner eine Variable zur Hauptaktivität der Neuzuwanderer. Einbindung in den Arbeitsmarkt, und gegebenenfalls auch das Studium an einer Universität, bringen Neuzuwanderer schnell mit der Aufnahmegesellschaft in Kontakt und dürften insofern entsprechende Orientierungen befördern, während dies bei Arbeitslosigkeit, Erziehungs- oder sonstiger häuslicher Tätigkeit weniger der Fall ist. Soziale Assimilation lässt sich über die Netzwerke starker oder schwacher Bindungen mit Mitgliedern der Aufnahmegesellschaft messen. Aufgrund der Situation der Neuzuwanderer konzentrieren wir uns auf starke Bindungen, wie sie sich in der jeweiligen Wohnsituation widerspiegeln; mit in Deutschland geborenen Personen zusammenzuwohnen, eröffnet unmittelbar Wege in die Aufnahmegesellschaft, während das Zusammenleben mit Polen oder Türken ethnische Orientierungen bekräftigen sollte. Um auch Gelegenheiten zu schwachen Bindungen zu berücksichtigen, fügen wir ferner eine Variable zur residenziellen Segregation ein, genauer: zur subjektiven Wahrnehmung des Anteils von Bewohnern aus dem eigenen Herkunftsland im jeweiligen Stadtviertel. Unsere Annahme ist, dass diejenigen, die in stark segregierten Stadtvierteln leben, eher ethnische Orientierungen entwickeln und leichter Zugang zu polnischen Kirchen oder türkischen Moscheen erhalten werden.

Das dritte, der Theorie symbolischer Grenzziehung entlehnte Argument prüfen wir durch Vergleich der zwei Modelle. Träfe das Argument zu, so wäre bei Türken mit einem

5 Das Basismodell enthält auch die Stadt des Interviews, um für eventuelle Selektivität der Befragten und nicht beobachtete Kontextfaktoren kontrollieren zu können. 
Tab. 1: Verteilung der Modellvariablen, polnische Katholiken und türkische Muslime (Mittelwerte). (Quelle: SCIP Projekt, eigene Berechnungen)

\begin{tabular}{lcc}
\hline & Polen & Türken \\
\hline Abhängige Variablen & & \\
Religiöse Partizipation vor Migration: Kirchgang oder Moscheebesuch & 3,40 & 2,47 \\
(1=nie; $7=$ wöchentlich) & & \\
Religiöse Praxis nach Migration: Gebet (1=nie; 6=täglich/mehrmals am Tag) & 3,50 & 2,68 \\
Unabhängige Variablen & & \\
Religiöse Partizipation vor Migration: Kirchgang oder Moscheebesuch & 4,75 & 4,01 \\
(1=nie; 7=wöchentlich) & & \\
Religiöse Praxis vor Migration: Gebet (1=nie; 6=täglich/mehrmals am Tag) & 3,79 & 3,19 \\
Geschlecht (weiblich) & 0,45 & 0,49 \\
Alter & 33 & 28 \\
Höchster Bildungsabschluss: Elementarstufe & 0,06 & 0,18 \\
Sekundarstufe & 0,71 & 0,46 \\
Tertiäre Stufe & 0,22 & 0,33 \\
Herkunftsregion (1=urban, 5=ländlich) & 2,58 & 1,79 \\
Anteil Polen/Türken in Stadtviertel & 2,04 & 2,41 \\
(1=(fast)keine; 5=(fast)alle) & & \\
Aufenthalt (Monate seit Immigration) & 8,62 & 7,37 \\
Migrationspläne: Verbleib in Deutschland & 0,23 & 0,42 \\
Pendeln & 0,23 & 0,20 \\
Remigration & 0,40 & 0,29 \\
Migration in Drittland & 0,05 & 0,04 \\
Hauptaktivität: Beschäftigung & 0,65 & 0,22 \\
Vollzeitbildung & 0,16 & 0,27 \\
Arbeitslos & 0,08 & 0,31 \\
Haushaltstätigkeit/Elternzeit & 0,08 & 0,13 \\
Sonstiges (Rente, Krankheit, Behinderung, k. A.) & 0,02 & 0,07 \\
Wohnsituation: lebt alleine & 0,20 & 0,15 \\
Mit in Deutschland Geborenen & 0,34 & 0,32 \\
Mit in Polen/Türkei Geborenen & 0,10 & 0,34 \\
Mit beiden & 0,36 & 0,20 \\
\hline & & \\
& &
\end{tabular}

positiven Effekt der Aufenthaltsdauer auf Religiosität zu rechnen, und zwar auch nach Kontrolle der Assimilationsvariablen.

Wir präsentieren die Ergebnisse beider Modelle separat für Polen und Türken, da sich so die im Zentrum des Interesses stehenden Gruppenunterschiede in den Effekten der unterschiedlichen Variablen am anschaulichsten zeigen lassen.

\section{Ergebnisse}

Aufschlussreich im Lichte aktueller Debatten über Zuwanderung sind zunächst allein schon die deskriptiven Befunde zu polnischen und türkischen Neuzuwanderern in 
Deutschland (Tab. 1). Beide Gruppen, jeweils im Schnitt acht Monate im Lande, teilen eine Reihe von Charakteristika. Es handelt sich um junge Menschen aus urbanen oder suburbanen Regionen in den Herkunftsländern (vor allem im Falle der Türken), die vergleichsweise hochgebildet sind. $71 \%$ der Polen und $46 \%$ der Türken geben als höchsten Bildungsabschluss die Sekundarstufe, weitere 22 bzw. $33 \%$ einen Hochschulabschluss an. Allerdings sind auch einige wichtige Unterschiede zwischen beiden Gruppen festzuhalten. So plant fast die Hälfte der türkischen Neuankömmlinge in Deutschland zu bleiben, während dies bei den Polen nur ein Viertel der Befragten tun. Ferner geben $65 \%$ Polen im Vergleich zu nur 22\% Türken ein Beschäftigungsverhältnis als wichtigste Tätigkeit an. Die Zahlen reflektieren insofern nochmals die oben geschilderten Migrationsgeschichten beider Gruppen.

Was die Religiosität angeht, so fällt für Polen und Türken gleichermaßen deren relativ hohes Niveau auf. Es ist allerdings zu beachten, dass die Religiosität, insbesondere bei den Türken, insgesamt unterhalb der Niveaus ähnlicher Altersgruppen in den Herkunftsländer liegen. Im ISSP beispielsweise beschreiben sich mehr als $70 \%$ der Polen und mehr als $80 \%$ der Türken als etwas, sehr und extrem religiös, während die entsprechenden Zahlen religiöser Selbsteinschätzung bei unseren Befragten bei 66,8\% bzw. 53,9\% liegen (nicht in Tabelle angegeben). ${ }^{6}$ Unbestreitbar ist aber, dass die Mittelwerte von Kirchgang oder Moscheebesuch und Gebet (Tab. 1) deutlich über denjenigen der deutschen Mehrheitsbevölkerung liegen.

Der Blick auf die Mittelwerte zeigt allerdings auch, dass religiöse Aktivitäten für beide Gruppen nach dem Migrationsereignis deutlich abnehmen, und zwar insbesondere für Kirchgang oder Moscheebesuch. Das unterschiedliche Ausmaß der Abnahme von Kirchgang oder Moscheebesuch im Vergleich zum Gebet bestätigt insoweit das Argument religiöser Opportunitätsstrukturen, das eine starke Abnahme vor allem für solche religiöse Praktiken prognostiziert, die auf Informationen über oder Zugang zu religiöser Infrastruktur angewiesen sind.

Insgesamt bekräftigen die deskriptiven Befunde, dass Migration für Religiosität zunächst ein disruptives Ereignis darstellt. Es scheint im Übrigen auch nicht so zu sein, dass die Religionsgemeinschaften für Neuzuwanderer die von Hirschman hervorgehobenen Ressourcen bereithalten. Von denjenigen, die überhaupt Kirchen oder Moscheen besuchen, geben, wie ergänzende (hier nicht dargestellte) Analysen zeigen, weniger als $10 \%$ an, Hilfestellung bei Wohnungs- oder Jobsuche erhalten zu haben.

Ein genaueres Bild der Abnahme der Religiosität nach dem Migrationsereignis im Gruppenvergleich bietet das Balkendiagramm in Abb. 1, das für beide Gruppen sämtliche Kombinationen religiöser Partizipation vor und nach der Migration darstellt. Wir beschränken uns auf Daten zu Kirchgang oder Moscheebesuch, weil diese nicht nur die größere Varianz aufweisen, sondern für die Untersuchung früher Integrationsmuster von besonderem inhaltlichem Interesse sind. Für die Zeit vor der Migration zeigt sich zunächst, dass katholische Polen häufiger in die Kirche gehen als muslimische Türken die Moschee

6 Daten aus den USA deuten daraufhin, dass solche Niveauunterschiede mit der Selektivität der Migranten in Verbindung stehen könnten; diejenigen, die sich zur Migration entscheiden, scheinen demnach etwas weniger stark in religiöse Gemeinschaften in den Herkunftsländern eingebettet zu sein (vgl. Alanezi und Sherkat 2008). 


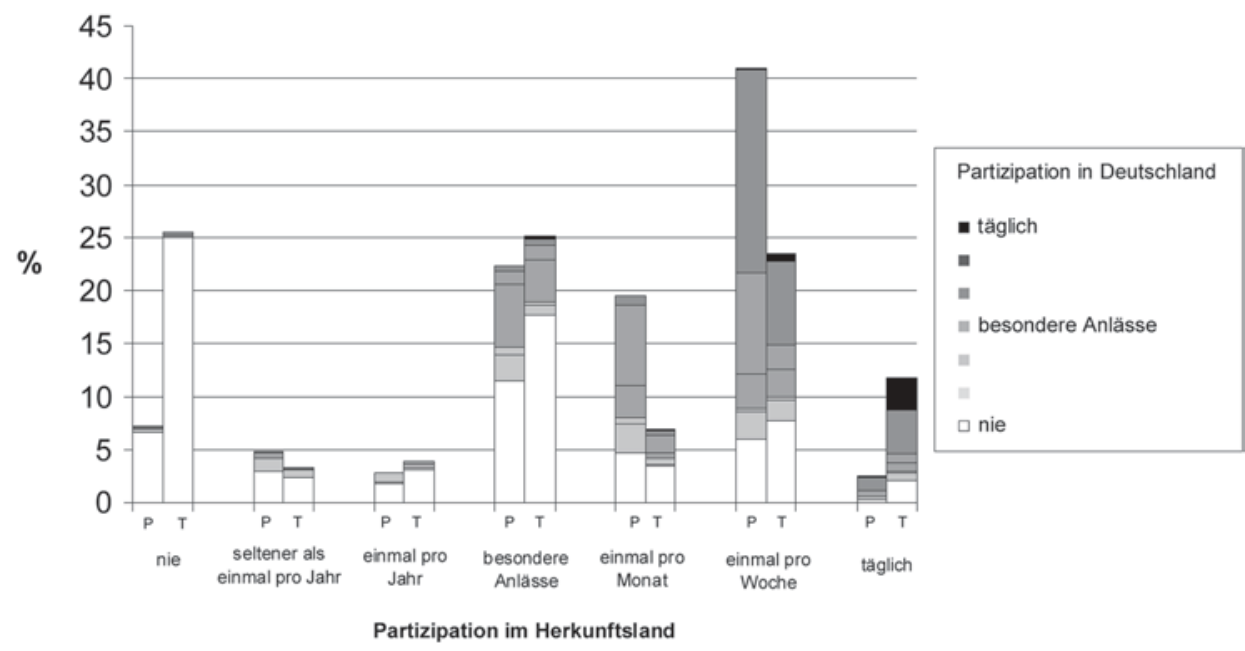

Abb. 1: Religiöse Partizipation polnischer Katholiken (P) und türkischer Muslime (T) in Deutschland, nach religiöser Partizipation im Herkunftsland

besuchen, was auf die bekannten Geschlechterunterschiede im Ausmaß des Moscheegangs zurückzuführen ist. Bemerkenswert ist, dass Türken hinsichtlich ihrer religiösen Praktiken deutlich polarisierter sind als Polen. Einerseits ist ein Viertel der türkischen Neuzuwanderer schon in der Türkei nie in die Moschee gegangen, während der entsprechende Anteil bei den Polen lediglich knapp über $5 \%$ liegt. Andererseits geben mehr als $10 \%$ der Türken, aber weniger als $5 \%$ der Polen an, täglich eine Moschee oder Kirche zu besuchen.

Für die Zeit nach der Migration zeigt Abb. 1, dass ein Anstieg religiöser Partizipation nur bei äußerst wenigen Polen und Türken zu beobachten ist, was nochmals unterstreicht, dass von einem religionsproduktiven Effekt des Migrationsereignisses nicht die Rede sein kann. Stabilität findet man entsprechend vor allem bei denen, die ohnehin nicht in die Kirche oder in die Moschee gehen. Von analytischem Interesse sind daher insbesondere die Muster der Abnahme religiöser Partizipation. Hier zeigt sich, dass viele Befragte, gleich wie oft sie im Herkunftsland die Kirche oder die Moschee besucht haben, angeben, sie würden dies in Deutschland gar nicht tun. Von den Türken beispielsweise, die in ihrer Heimat wöchentlich in die Moschee gegangen sind, bleibt ein ganzes Drittel in Deutschland dem Moscheeleben fern. Es liegt auf der Hand, dass solche extremen und abrupten Veränderungen auf einen Mangel an Zugang zu religiöser Infrastruktur zurückzuführen sind, die für den Aufrechterhalt religiöser Routinen erforderlich ist.

Das Argument der Opportunitätsstrukturen wird zudem im Gruppenvergleich unterstützt. Zwar zeigen die SCIP-Daten (hier nicht dargestellt), dass regelmäßig religiös partizipierende Polen und Türken sich gleichermaßen in ethnisch homogenen Kirchen oder Moscheen finden. Dennoch ist anzunehmen, dass Polen angesichts der institutionellen Präsenz der katholischen Kirche in deutschen Städten etwas günstigere Opportunitäten für religiöse Partizipation vorfinden; polnischsprachige Messen gehören in Städten wie Köln oder München schließlich inzwischen zum regulären Angebot der katholischen Bis- 
Tab. 2: Welche Faktoren beeinflussen die Stabilität religiöser Partizipation? Polnische Katholiken und türkische Muslime (lineare Regression, nicht-standardisierte Koeffizienten)

Abhängige Variable: Religiöse Partizipation (Kirchgang/Moscheebesuch) nach Migration (7-Punkte Skala)

\begin{tabular}{|c|c|c|c|c|}
\hline & \multicolumn{2}{|c|}{ Polen $(N=1327)$} & \multicolumn{2}{|c|}{ Türken $(N=985)$} \\
\hline & Modell I & Modell II & Modell I & Modell II \\
\hline Religiöse Partizipation vor Migration & $0,75 * * *$ & $0,73 * * *$ & $0,53 * * *$ & $0,50 * * *$ \\
\hline Weiblich & $0,16^{*}$ & $0,24 *$ & $-0,61 * * *$ & $-0,66 * * *$ \\
\hline Alter & $0,02 * *$ & $0,02 * *$ & 0,00 & $-0,01$ \\
\hline \multicolumn{5}{|c|}{ Höchster Bildungsabschluss: Elementarstufe } \\
\hline Sekundarstufe & $-0,01$ & $-0,04$ & $-0,17$ & $-0,20$ \\
\hline Tertiäre Stufe & 0,13 & 0,13 & $-0,16$ & $-0,17$ \\
\hline Ländlicher Hintergrund & 0,04 & 0,05 & 0,03 & 0,01 \\
\hline Anteil Polen/Türken in Stadtviertel & & 0,03 & & 0,03 \\
\hline Aufenthalt (Monate seit Immigration) & $-0,01$ & 0,00 & $0,04 * *$ & $0,03 * *$ \\
\hline \multicolumn{5}{|l|}{ Migrationspläne: Verbleib in Deutschland } \\
\hline Pendeln & & 0,11 & & 0,04 \\
\hline Remigration & & $-0,02$ & & $0,41 * *$ \\
\hline Migration in Drittland & & 0,04 & & 0,26 \\
\hline \multicolumn{5}{|l|}{ Hauptaktivität: Beschäftigung } \\
\hline Vollzeitbildung & & $0,43 * *$ & & $0,35 * *$ \\
\hline Arbeitslos & & 0,11 & & $-0,06$ \\
\hline Haushaltstätigkeit/Elternzeit & & $-0,07$ & & $-0,28$ \\
\hline $\begin{array}{l}\text { Sonstiges (Rente, Krankheit, } \\
\text { Behinderung, k. A.) }\end{array}$ & & 0,41 & & 0,11 \\
\hline \multicolumn{5}{|c|}{ Wohnsituation: Lebt mit in Deutschland Geborenen } \\
\hline Mit in Polen/Türkei Geborenen & & $0,56^{* *}$ & & $-0,02$ \\
\hline Alleine & & $0,58 * *$ & & $-0,13$ \\
\hline Mit beiden & & $0,45 * *$ & & 0,15 \\
\hline Konstante & $-0,933 * *$ & $-1,911 * * *$ & $1,160 * *$ & $1,262 * *$ \\
\hline $\mathrm{R}^{2}$ & 0,327 & 0,353 & 0,371 & 0,390 \\
\hline
\end{tabular}

$* \mathrm{p}<0,10, * * \mathrm{p}<0,05, * * * \mathrm{p}<0,001$; in allen Modellen wird Stadt des Interviews kontrolliert

tümer. Und tatsächlich ist der Kirchgang bei den Polen entsprechend weniger rückläufig als bei den türkischen Neuzuwanderern.

Um die theoretischen Argumente zur religiösen Dynamik in der Frühphase der Integration zu prüfen, wenden wir uns nunmehr der multivariaten Betrachtung des Kirchgangs oder Moscheebesuchs zu. Das Basismodell (Tab. 2, Basismodell für beide Gruppen) beinhaltet religiöse Partizipation vor der Auswanderung, sozio-demographische Variablen, Aufenthaltsdauer und, in der Tabelle nicht abgebildet, die Stadt, in der das Interview geführt wurde. Es erstaunt nicht, dass religiöse Partizipation vor und nach der Migration bei beiden Gruppen hochgradig miteinander korreliert, wenngleich die Effekte für Polen $(b=0,75)$ etwas stärker sind als diejenigen für die Türken $(b=0,53)$. Davon abgesehen unterscheiden sich die Muster religiöser Partizipation allerdings deutlich zwischen beiden Gruppen. Türkinnen besuchen, auch nach Kontrolle vorheriger religiöser Gewohn- 
heiten(!), in Deutschland weniger häufig die Moschee, während es bei den polnischen Neuzuwanderern vor allem ältere Frauen sind, die nach wie vor häufig in die Kirche gehen. Damit bleibt Religiosität im Wesentlichen bei den sozio-demographischen Gruppen stabil, die ohnehin religiöser sind. So zeigen separate (hier nicht dargestellte) Analysen, dass Religiosität (über einen additiven Index gemessen) insgesamt bei den Polen mit Alter, weiblichem Geschlecht und ländlicher Herkunft korreliert ist, während bei den Türken die jüngeren und männlichen Befragten religiöser sind.

Aufschlussreich ist nun, dass die Aufenthaltsdauer bei den Türken religiöse Partizipation zu stabilisieren scheint $(b=0,04)$, nicht aber bei Polen. Zwar ist der Einbruch religiöser Partizipation bei den Türken, wie gesagt, zunächst stärker, aber er scheint bei ihnen von einem Prozess der allmählichen Reorganisation religiöser Praktiken gefolgt zu sein, der bei den Polen so nicht zu beobachten ist.

Um das assimilationstheoretische Argument und das Argument symbolischer Grenzdynamiken genauer zu testen, werden im erweiterten Modell die Variablen sozialer und struktureller Assimilation ergänzt. Während die sozio-demographischen Effekte in diesen Modellen stabil bleiben, treten Gruppenunterschiede in den Mustern religiöser Partizipation noch deutlicher hervor. Bei den Polen scheinen Bleibeabsichten zwar keine statistisch signifikanten Effekte zu haben, die Effekte sozialer Assimilation weisen aber in die von der Assimilationstheorie her erwartete Richtung. Diejenigen Polen, die ausschließlich mit Deutschen zusammenwohnen, gehen tendenziell seltener in die Kirche als diejenigen, die ausschließlich oder teilweise mit Polen zusammenwohnen.

Dies stellt sich bei den Türken deutlich anders dar. Zwar bleibt religiöse Partizipation bei Befragten mit eindeutigen Rückkehrabsichten stabiler als bei denen, die planen, in Deutschland zu bleiben $(b=0,41)$. Die Wohnsituation, also das Zusammenleben mit Deutschen oder mit anderen türkischen Migranten, hat aber keinen statistisch signifikanten Effekt auf religiöse Partizipation, ebenso wenig die ethnische Segregation der Nachbarschaft. ${ }^{7}$ Vor allem aber bleibt der positive Effekt der Aufenthaltsdauer auch nach Kontrolle der Assimilationsvariablen stabil. Dieser Befund stützt unser drittes Argument zu religiösen Grenzdynamiken, die von sozialer Assimilation entkoppelt sind. Anders als katholische Polen, die eine bestehende religiöse Infrastruktur vorfinden, erfahren Türken zwar einen schärferen Rückgang religiöser Partizipation; sie wenden sich im Laufe der Zeit aber eher wieder dem Moscheeleben zu als die Polen.

Weniger eindeutig fallen die Befunde für die Effekte struktureller Assimilation, also der Einbindung in den Arbeitsmarkt, aus. Interessant ist allerdings, dass innerhalb beider Gruppen vor allem diejenigen, die im Bildungssystem integriert sind, also typischerweise Studierende an Universitäten oder Fachhochschulen, häufiger in die Kirche oder Moschee gehen. Dies lässt sich dahingehend interpretieren, dass Studierende aufgrund diverser

7 Unsere Daten gestatten es nicht auszuschließen, dass die in Deutschland geborenen Personen, mit denen manche türkische Neuzuwanderer zusammenleben, einen türkischen Migrationshintergrund haben, also etwa zur zweiten Generation gehören. Wir haben deshalb alternative Berechnungen durchgeführt und die ethnische Zusammensetzung des engsten Freundschaftsnetzwerks untersucht. Dass der türkische Hintergrund (nicht türkischer Geburtsort) der besten drei Freunde keine Effekte auf religiöse Partizipation hat, bekräftigt uns in der Einschätzung, dass soziale Assimilation und Religiosität bei den türkischen Neuzuwanderern voneinander unabhängig sind. 
Tab. 3: Welche Faktoren beeinflussen die Stabilität privater religiöser Praxis? Polnische Katholiken und türkische Muslime (lineare Regression, nicht-standardisierte Koeffizienten)

Abhängige Variable: Religiöse Praxis (Gebet) nach Migration (6-Punkte Skala)

\begin{tabular}{|c|c|c|c|c|}
\hline & \multicolumn{2}{|c|}{ Polen $(N=1276)$} & \multicolumn{2}{|c|}{ Türken $(N=966)$} \\
\hline & Modell I & Modell II & Modell I & Modell II \\
\hline Religiöse Praxis vor Migration & $0,90 * * *$ & $0,90 * * *$ & $0,77 * * *$ & $0,76^{* * *}$ \\
\hline Weiblich & $0,27 * * *$ & $0,34 * * *$ & $0,16^{*}$ & 0,09 \\
\hline Alter & 0,00 & 0,00 & 0,01 & 0,00 \\
\hline \multicolumn{5}{|l|}{ Höchster Bildungsabschluss: Elementarstufe } \\
\hline Sekundarstufe & $-0,03$ & $-0,01$ & $-0,14$ & $-0,13$ \\
\hline Tertiäre Stufe & 0,06 & 0,09 & $-0,15$ & $-0,16$ \\
\hline Ländlicher Hintergrund & 0,04 & 0,04 & $-0,02$ & $-0,03$ \\
\hline Anteil Polen/Türken in Stadtviertel & & $-0,06^{*}$ & & 0,02 \\
\hline Aufenthalt (Monate seit Immigration) & 0,00 & $-0,01$ & $0,02 * *$ & $0,02 * *$ \\
\hline \multicolumn{5}{|l|}{ Migrationspläne: Verbleib in Deutschland } \\
\hline Pendeln & & 0,08 & & 0,05 \\
\hline Remigration & & 0,06 & & $0,23 * *$ \\
\hline Migration in Drittland & & 0,16 & & 0,24 \\
\hline \multicolumn{5}{|l|}{ Hauptaktivität: Beschäftigung } \\
\hline Vollzeitbildung & & $-0,15^{*}$ & & $-0,15$ \\
\hline Arbeitslos & & 0,11 & & 0,06 \\
\hline Haushaltstätigkeit/Elternzeit & & $-0,15$ & & $-0,01$ \\
\hline $\begin{array}{l}\text { Sonstiges } \\
\text { (Rente, Krankheit, Behinderung, k. A.) }\end{array}$ & & $0,36 * *$ & & 0,15 \\
\hline \multicolumn{5}{|c|}{ Wohnsituation: Lebt mit in Deutschland Geborenen } \\
\hline Mit in Polen/Türkei Geborenen & & $0,31 * *$ & & 0,10 \\
\hline Alleine & & $0,29 * *$ & & 0,13 \\
\hline Mit beiden & & $0,32 * *$ & & $0,32 * *$ \\
\hline Konstante & $-0,442 * *$ & $-0,587 * *$ & $-0,168$ & $-0,246$ \\
\hline $\mathrm{R}^{2}$ & 0,741 & 0,745 & 0,619 & 0,620 \\
\hline
\end{tabular}

$* \mathrm{p}<0,10, * * \mathrm{p}<0,05, * * * \mathrm{p}<0,001$; in allen Modellen wird Stadt des Interviews kontrolliert

katholischer und muslimischer Campus-Gruppen im Vergleich zu anderen Neuzuwanderern eher über (Wissen über) Gelegenheiten religiöser Partizipation verfügen (Tab. 3).

Die Einsichten der bisherigen Analyse erhärten sich, wenn wir uns der Gebetshäufigkeit als abhängiger Variable zuwenden. Nochmals findet das Opportunitätenargument Bestätigung, denn die Gebetshäufigkeit geht weitaus weniger drastisch zurück als der Kirchgang oder Moscheebesuch. Für beide Gruppen ist Gebetshäufigkeit vor und nach der Migration entsprechend stärker korreliert, wobei die Effekte bei Türken erneut schwächer sind als bei Polen. Interessant sind hier wiederum die geschlechtsspezifischen Muster. Anders als beim Moscheebesuch sind es nämlich die türkische Frauen, die eher zur Aufrechterhaltung ihrer Gebetspraxis neigen. Dies deutet darauf hin, dass der rückläufige Moscheebesuch bei Frauen auf besondere Merkmale der islamischen Organisationen in Deutschland zurückzuführen ist. Diese Interpretation wird auch durch weitere (hier nicht dargestellte) Analysen bestätigt, wonach das Kopftuchtragen weitgehend stabil bleibt; 
ein Drittel der Befragten gibt an, das Kopftuch in der Türkei immer, zwei Drittel, es nie getragen zu haben, und dies ändert sich auch nach der Migration nicht.

Bemerkenswert ist vor allem aber, dass die Aufenthaltsdauer erneut einen positiven Effekt auf die private religiöse Praxis bei den Türken, nicht aber bei Polen hat. Die Modelle bestätigen grosso modo die für den Gottesdienstbesuch geschilderten Befunde. Bei den Polen wirkt sich soziale Assimilation, gemessen über das Zusammenleben mit Deutschen, negativ auf Religiosität aus. Bei den Türken sind Rückkehrabsichten positiv mit Religiosität verbunden, während soziale Assimilation wiederum keine Effekte aufweist. Die Effekte der Aufenthaltsdauer bleiben bei den Türken stabil, was das Argument unterstützt, dass bei Neuzuwanderern aus der Türkei religiöse Routinen allmählich wieder stabilisiert werden. Strukturelle Assimilation ist bei beiden Gruppen ohne Einfluss auf die Religiosität. Zusätzlich zeigt sich, dass eine enge Einbindung ins Bildungssystem zwar wie oben dargestellt die gemeinschaftlichen religiösen Aktivitäten fördert, nicht aber in positivem Zusammenhang mit der Gebetshäufigkeit nach der Migration steht.

\section{Diskussion und Fazit}

In diesem Artikel haben wir Religiosität nach dem Migrationsereignis bei Neuzuwanderern aus Polen und der Türkei in Deutschland untersucht. Es ist eine der ersten Studien, die außerhalb des nordamerikanischen Kontextes den Einfluss des Migrationsereignisses auf Religiosität auf Basis quantitativ erhobener Daten analysiert. Der Artikel leistet einen Beitrag zur bisherigen Forschungsliteratur, indem er Dynamiken religiösen Wandel in der Frühphase der Integration im westeuropäischen Kontext und im Gruppenvergleich betrachtet.

Unsere Analysen bestätigen die wichtigsten in der Literatur anzutreffenden theoretischen Argumente zumindest teilweise. Eindeutig zeigt sich zunächst, dass das Migrationsereignis als solches auch in Europa keineswegs die religionsvitalisierende Erfahrung darstellt, als die es gelegentlich porträtiert wird. Vielmehr folgt ihm, ähnlich wie in Nordamerika, zunächst ein Einbruch religiöser Routinen. Das Argument, wonach dieser Einbruch zumindest teilweise auf fehlenden Zugang zu religiösen Opportunitäten zurückgeht, ließ sich weitgehend erhärten. Der Rückgang an Religiosität betraf bei beiden Gruppen vor allem die religiöse Partizipation (Kirchgang oder Moscheebesuch), weniger indessen die private religiöse Praxis (Gebet), und er fiel bei muslimischen Türken drastischer aus als bei katholischen Polen. ${ }^{8}$

Die entscheidende Frage ist allerdings, ob solche anfänglichen Einbrüche religiöser Routinen den Beginn längerfristiger Anpassungsprozesse an eine säkulare Aufnahmegesellschaft darstellen oder ob ihnen ein Prozess religiöser Re-Organisation folgt. Für polnische Neuzuwanderer stellte sich heraus, dass Migranten mit Bindungen in die deutsche Mehrheitsgesellschaft einen stärkeren Rückgang an Religiosität erfahren als diejenigen,

8 Was die Unterschiede von Kirchgang und Gebetshäufigkeit angeht, kommt eine auf SCIP-Daten basierende Untersuchung in den Niederlanden inzwischen zu demselben Ergebnis (van Tubergen 2013); die Gruppenunterschiede fallen allerdings anders aus, was Anlass zur stärkeren Berücksichtigung länderspezifischer Integrationskontexte gibt. 
die in ethnische Netzwerken eingebunden sind. Es gibt bei ihnen auch keinerlei Anzeichen dafür, dass religiöse Praktiken sich nach den ersten Monaten wieder intensivieren. Genau dies scheint indessen bei den türkischen Neuzuwanderern der Fall zu sein. Je länger sie in Deutschland sind, desto mehr kehren zumindest diejenigen, die nicht schon in der Türkei areligiös waren, zu ihren religiösen Routinen zurück. Dieser Prozess religiöser Re-Organisation, den die klassische Assimilationstheorie nur schwer zu erfassen vermag, verläuft relativ unabhängig von sozialer und struktureller Assimilation. Einiges deutet vielmehr darauf hin, dass saliente symbolische Grenzen gegenüber dem Islam in Deutschland dafür eine wichtige Rolle spielen; sie erschweren graduelle Akkulturation und tragen damit zu einer größeren religiösen Stabilität bei, die sich im Übrigen auch im intergenerationalen Vergleich nachweisen lässt (vgl. Diehl und Koenig 2009).

Es liegt auf der Hand, dass unsere Analyse eine Reihe von Fragen offenlässt. Verglichen mit qualitativen Studien geben unsere Daten keinen Aufschluss darüber, welchen Religionsgemeinschaften sich Neuzuwanderer im Einzelnen zuwenden; bei den Türken beispielsweise wäre von Interesse, welche Bindungskraft die von der DIBIT oder von Milli Görüs organisierten Moscheen unter Neuzuwanderern entfalten, um nur die zwei prominentesten Varianten des türkischen Islam in Deutschland zu erwähnen (Schiffauer 2009). Weiter bleibt unbefriedigend, dass der religiöse Wandel nach dem Migrationsereignis lediglich mit Hilfe retrospektiver Fragen zu Kirchgang oder Moscheebesuch und Gebet sowie durch den Effekt der Aufenthaltsdauer gemessen wird, und dass durch die zeitinvariable Messung struktureller und sozialer Assimilation die frühe Integrationsdynamik allenfalls ansatzweise erfasst wird. Erst die Daten der zweiten Erhebungswelle werden es gestatten, diese Beschränkungen zu überwinden und robustere Ergebnisse über die Frühphase religiöser Adaptation zu liefern.

Doch trotz dieser Einschränkungen bieten unsere Analysen erste komparative Einsichten in jene Frühphase religiöser Adaptation im westeuropäischen Kontext, in der Veränderungen individueller Religiosität auf verschiedene, komplex miteinander verschränkte Mechanismen zurückzuführen sind. Die empirischen Befunde legen insbesondere nahe, dass die klassische Assimilationstheorie um solche Argumente ergänzt werden muss, die auch die Dynamik religiöser Re-Organisation bei Zuwanderergruppen erfassen, die sich scharf gezogenen symbolischen Grenzen gegenüber sehen. Sollten sich die empirischen Hinweise auf Prozesse religiöser Re-Organisation bei Zuwanderern aus nicht-christlichen Herkunftsländern in Europa weiter verdichten, so hätte dies weitreichende Implikationen. Es würde weitere Evidenz für die Stabilisierung religiös-ethnischer Identitäten bei Einwanderern geben (vgl. auch Brubaker 2013) und damit bestätigen, dass öffentlich sichtbare religiöse Diversität ein (zunächst) dauerhaftes Merkmal europäischer Einwanderungsgesellschaften bleibt. Es ist insofern damit zu rechnen, dass sie allen sonstigen Säkularisierungstendenzen zum Trotz weiterhin ein Gegenstand öffentlicher Auseinandersetzungen sein wird.

Danksagung: Die diesem Artikel zu Grunde liegende Forschung wurde finanziell durch das NORFACE Forschungsprogramm „Migration in Europe - Social, Economic, Cultural and Policy Dynamics“ gefördert. Wir bedanken uns bei Phillip Connor, Christof Wolf sowie den Teilnehmern der Autorentagung des Sonderhefts für kritische Kommentare und hilfreiche Anregungen. Eine kürzere Version dieses Beitrags wurde auf dem 36. Kongress der Deutschen Gesellschaft für Soziologie 
präsentiert und ist in englischer Sprache in der Zeitschrift International Migration 51/3 (2013) erschienen.

\section{Literatur}

Abramson, Harold J. 1979. Migrants and cultural diversity: On ethnicity and religion in society. Social Compass 26:5-29.

Adida, Claire L., David Laitin und Marie-Anne Valfort. 2010. Identifying barriers to Muslim integration in France. Proceedings of the National Academy of Sciences of the United States of America 107:22384-22390.

Akresh, Ilana Redstone. 2011. Immigrants' religious participation in the United States. Ethnic and Racial Studies 34:643-661.

Alanezi, Fawaz, und Darren E. Sherkat. 2008. The religious participation of US immigrants: exploring contextual and individual influences. Social Science Research 37:844-855.

Alba, Richard. 2005. Bright vs. blurred boundaries: Second-generation assimilation and exclusion in France, Germany, and the United States. Ethnic and Racial Studies 28:20-49.

Alba, Richard, und Victor Nee. 2003. Remaking the American mainstream: Assimilation and contemporary immigration. Cambridge: Harvard University Press.

Alba, Richard, Albert J. Raboteau und Josh DeWind. Hrsg. 2009. Immigration and religion in America. Comparative and historical perspectives. New York: New York University Press.

Aleksynska, Mariya, und Yann Algan. 2010. Economic and cultural assimilation and integration of immigrants in Europe. CEPII Working Paper 29.

Berry, John W. 1997. Immigration, acculturation, and adaptation. Applied Psychology. An International Review 46:5-68.

Bisin, Alberto, Thierry Verdier, Eleanora Patacchini und Yves Zenou. 2008. Are Muslim immigrants different in terms of cultural integration? Journal of the European Economic Association 6:445-456.

Blohm, Michael, und Martina Wasmer. 2008. Einstellungen und Kontakte zu Ausländern. In Datenreport 2008, Hrsg. Statistisches Bundesamt Deutschland, 208-214. Bonn: Statistisches Bundesamt.

Breton, Raymond. 1964. Institutional completeness of ethnic communities and the personal relations of immigrants. American Journal of Sociology 70:193-205.

Breton, Raymond. 2012. Different gods. Integrating non-christian minorities into a primarily christian society. Montreal: McGill-Queen's University Press.

Brubaker, Rogers. 2013. Language, religion and the politics of difference. Nations and Nationalism 19:1-20.

Bruce, Steve. 2011. Secularization. In defence of an unfashionable theory. Oxford: Oxford University Press.

Bundesministerium für Inneres. Hrsg. 2010. Migrationsbericht des Bundesamtes für Migration und Flüchtlinge im Auftrag der Bundesregierung (Migrationsbericht 2008). Berlin: Bundesministerium für Inneres.

Cadge, Wendy, und Elaine Howard Ecklund. 2006. Religious service attendance among immigrants. Evidence from the new immigrant survey-pilot. American Behavioral Scientist 49:1574-1595.

Cadge, Wendy, und Elaine Howard Ecklund. 2007. Immigration and religion. Annual Review of Sociology 33:359-379.

Casanova, José. 1994. Public religions in the modern world. Chicago: Chicago University Press.

Connor, Phillip. 2008. Increase or decrease? The impact of international migratory event on immigrant religious participation. Journal for the Scientific Study of Religion 47:243-257.

Connor, Phillip. 2009a. International migration and religious participation: The mediating impact of individual and contextual effects. Sociological Forum 24:779-803. 
Connor, Phillip. 2009b. Immigrant religiosity in Canada: Multiple trajectories. Journal of International Migration and Integration 10:159-175.

Connor, Phillip. 2010. Contexts of immigrant receptivity and immigrant religious outcomes: The case of Muslims in Western Europe. Ethnic and Racial Studies 33:376-403.

Connor, Phillip, und Matthias Koenig. 2013. Bridge and barrier - religion and immigrant occupational attainment across integration contexts. International Migration Review 36:3-38.

Diehl, Claudia, und Matthias Koenig. 2009. Religiosität türkischer Migranten im Generationenverlauf - ein Befund und einige Erklärungsversuche. Zeitschrift für Soziologie 38:300-319.

Esser, Hartmut. 2006. Does the ,new“ immigration require a ,new“ theory of intergenerational integration? International Migration Review 38:1126-1159.

Fleischmann, Fenella, und Karen Phalet. 2012. Integration and religiosity among the Turkish second generation in Europe: A comparative analysis across four capital cities. Ethnic and Racial Studies 35:320-341.

Foner, Nancy, und Richard Alba. 2008. Immigrant religion in the U.S. and Western Europe: Bridge or barrier to inclusion? International Migration Review 42:360-392.

Glock, Charles Y. 1962. On the study of religious commitment. Religious Education 57:98-110.

Gordon, Milton. 1964. Assimilation in American life: The role of race, religion, and national origins. New York: Oxford University Press.

Green, Nancy L. 2002. Religion et ethnicité. De la comparaison spatiale et temporelle. Annales. Histoire, Sciences Sociales 57:127-144.

Hagan, Jacqueline, und Helen Rose Ebaugh. 2003. Calling upon the sacred: Migrants' use of religion in the migration process. International Migration Review 37:1145-1162.

Hammond, Phillip E., und Kee Warner. 1993. Religion and ethnicity in late-twentieth-century America. The ANNALS of the American Academy of Political and Social Science 527:55-66.

Hans, S. 2010. Assimilation oder Segregation? Anpassungsprozesse von Einwanderern in Deutschland. Wiesbaden: VS Verlag für Sozialwissenschaften.

Hardin, R. 2001. Social Identity. International Encyclopedia of the Social and Behavioral Sciences, Hrsg. Neil J. Smelser and Paul B. Baltes, S. 7166-7160. Oxford : Elsevier.

Herbergs, Will. 1956. Protestant, Catholic, Jew. An Essay in American Religious Sociology. New York: Doubleday \& Company.

Hirschman, Charles. 2004. The role of religion in the origins and adaptation of immigrant groups in the United States. International Migration Review 38:1206-1233.

Jacob, Konstanze, und Frank Kalter. 2013. Intergenerational change in religious salience among immigrant families in four European countries. International Migration 51:38-56.

Koenig, Matthias. 2007. Europäisierung von Religionspolitik. Zur institutionellen Umwelt der Anerkennungskämpfe muslimischer Migranten. In Soziale Welt - Sonderheft „Islam in Europa “, Hrsg. Monika Wohlrab-Sahr und Levent Tezcan, 347-368. Baden-Baden: Nomos.

Laurence, Jonathan. 2012. The emancipation of Europe's Muslims. The state's role in minority integration. Princeton: Princeton University Press.

Levitt, Peggy. 2003. You know, Abraham really was the first immigrant: Religion and transnational migration. International Migration Review 37:847-873.

Maliepaard, Mieke, Marcel Lubbers und Merove Gijsberts. 2010. Generational differences in ethnic and religious attachment and their interrelation: a study among Muslim minorities in the Netherlands. Ethnic and Racial Studies 33:451-471.

Massey, Douglas S., und Monica Espinoza Higgins. 2011. The effect of immigration on religious belief and practice: A theologizing or alienating experiences. Social Science Research 40:1371-1389.

Mitchell, Claire. 2006. The religious content of ethnic identities. Sociology 40:1135-1152.

Mol, Hans. 1979. Theory and data on the religious behaviour of migrants. Social Compass 26:31-39.

Mullins, Mark. 1987. The life-cycle of ethnic churches in sociological perspective. Japanese Journal of Religious Studies 14:321-334. 
Norris, Pippa, and Ronald Inglehart. 2004. Sacred and secular. Religion and politics worldwide. Cambridge: Cambridge University Press.

Pew Research Centre. 2012. Faith on the move. The religious affiliation of international migration. Washington: Pew Research Centre.

Phalet, Karen, Merove Gijsberts und Louk Hagendoorn. 2008. Migration and religion: Testing the limits of secularisation among Turkish and Moroccan Muslims in the Netherlands 1998-2005. In Migration und Integration, Sonderheft 48 Kölner Zeitschrift für Soziologie und Sozialpsychologie, Hrsg. Frank Kalter, 412-436. Wiesbaden: VS Verlag für Sozialwissenschaften.

Phalet, Karen, und Ute Schönpflug. 2001. Intergenerational transmission in Turkish immigrant families: Parental collectivism, achievement values and gender differences. Journal of Comparative Family Studies 32:489-504.

Schiffauer, Werner. 2004. Vom Exil- zum Diaspora-Islam. Muslimische Identitäten in Europa. Soziale Welt 55:347-368.

Schiffauer, Werner. 2009. Nach dem Islamismus. Frankfurt a. M.: Suhrkamp.

Smith, Timothy. 1978. Religion and ethnicity in America. American Historical Review 83:1155-1185.

Smits, Fransje, Stijn Ruiter und Frank van Tubergen. 2010. Religious practices among Islamic immigrants: Moroccans and Turkish men in Belgium. Journal for the Scientific Study of Religion 49:247-263.

Statham, Paul, Ruud Koopmans, Marco Giugni und Florence Passy. 2005. Resilient or adaptable Islam? Multiculturalism, religion and migrants' claims-making for group demands in Britain, the Netherlands and France. Ethnicities 5:427-459.

Stolz, Jörg. 2009. Explaining religiosity: Towards a unified theoretical model. British Journal of Political Science 60:345-376.

Stolz, Jörg. 2010. The ,social integration" of religious groups in society: A social mechanisms approach. Annual Review of the Sociology of Religion 2:85-116.

Tezcan, Levent. 2003. Das Islamische in den Studien zu Muslimen in Deutschland. Zeitschrift für Soziologie 32:237-261.

Tribalat, Michèle. 1995. Faire France. Une enquête sur les immigrés et leurs enfants. Paris: La Découverte.

Van Tubergen, Frank. 2013. Religious change of new immigrants in the Netherlands: The event of migration. Social Science Research 42:715-725.

Van Tubergen, Frank, und Jorunn I. Sindradottir. 2011. The religiosity of immigrants in Europe: A cross-national study. Journal for the Scientific Study of Religion 50:272-288.

Voas, David. 2009. The rise and fall of fuzzy fidelity in Europe. European Sociological Review 25:155-168.

Voas, David, und Fenella Fleischmann. 2012. Islam moves west: Religious change in the first and second generations. Annual Review of Sociology 38:525-545.

Yavuz, M. Hakan. 2003. Islamic political identity in Turkey. Oxford: Oxford University Press.

Zubrzycki, Geneviève. 2006. The crosses of Auschwitz: Nationalism and religion in post-communist Poland. Chicago: University of Chicago Press.

Claudia Diehl, 1968, Prof. Dr., Lehrstuhl für Mikrosoziologie an der Universität Konstanz. Forschungsgebiete: Migrations- und Integrationsprozesse. Ausgewählte Veröffentlichungen: Die Entscheidung zur Einbürgerung: Optionen, Anreize und identifikative Aspekte. Kölner Zeitschrift für Soziologie und Sozialpsychologie, Sonderheft 48, 2008 (mit M. Blohm); Religiosität türkischer Migranten im Generationenverlauf: ein Befund und einige Erklärungsversuche. Zeitschrift für Soziologie 38, 2009 (mit M. Koenig). 
Matthias Koenig, 1971, Prof. Dr. phil., Institut für Soziologie, Georg-August-Universität Göttingen. Forschungsgebiete: Religionssoziologie, historisch-komparative Soziologie, soziologische Theorie. Veröffentlichungen u. a.: Religionskontroversen in Frankreich und Deutschland. Hamburg 2008 (Hrsg. mit J.-P. Willaime); International migration and the governance of religious diversity. Montreal, Kanada 2009 (Hrsg. mit P. Bramadat); Jenseits des Säkularisierungsparadigmas? Eine Auseinandersetzung mit Charles Taylor. Kölner Zeitschrift für Soziologie und Sozialpsychologie 63, 2012. 